\title{
BBU AND CORKSCREW GROWTH PREDICTIONS FOR THE DARHT SECOND AXIS ACCELERATOR *
}

\author{
Yu-Jiuan Chen, LLNL, Livermore, CA 94550, U.S.A. \\ William M. Fawley, Lawrence Berkeley National Laboratory, Berkeley, CA 94720, U.S.A.
}

\begin{abstract}
The second axis accelerator of the Dual Axis Radiographic Hydrodynamic Test (DARHT-II) facility will produce a 2-kA, 20-MeV, 2- $\mu$ s output electron beam with a design goal of less than $1000 \pi \mathrm{mm}$-mrad normalized transverse emittance [1]. In order to meet this goal, both the beam breakup instability (BBU) and transverse "corkscrew" motion (due to chromatic phase advance) must be limited in growth. Using data from recent experimental measurements of the transverse impedance of actual DARHT-II accelerator cells by Briggs et al. [2], we have used the LLNL BREAKUP code to predict BBU and corkscrew growth in DARHT-II. The results suggest that BBU growth should not seriously degrade the final achievable spot size at the $\mathrm{x}$-ray converter, presuming the initial excitation level is of the order 100 microns or smaller. For control of corkscrew growth, a major concern is the number of "tuning" shots needed to utilize effectively the "tuning-V" algorithm [3]. Presuming that the solenoid magnet alignment falls within spec, we believe that possibly as few as 50-100 shots will be necessary to set the dipole corrector magnet currents. We give some specific examples of tune determination for a hypothetical set of alignment errors.
\end{abstract}

\section{INTRODUCTION}

At present the DARHT-II facility is in the last phases of construction and will be complete sometime in 2002 . The primary function of this facility is to produce high quality $\mathrm{x}$-ray radiographs for nonnuclear experiments designed to measure the many complex, dynamic aspects of a nuclear weapon primary, or pit, during its implosion phase $[1,4]$. Toward this end, there is a design goal for the output emittance of the $2-\mathrm{kA}, 20 \mathrm{MeV}, 2-\mu$ s electron beam pulse to be $1000 \pi \mathrm{mm}$-mrad or better. Much of the last couple years in beam dynamics portion of the projects has been spent in optimizing various parts of the transport lattice, from the injector optics at the front end down to final accelerator cell solenoids to help ensure that the beam quality will remain of sufficiently high quality. There are two major concerns within the majority of the accelerator lattice: the beam breakup instability (BBU) and transverse "corkscrew" motion. The former arises from the transverse resistive impedance of the induction cell accelerating gap regions and has an exponential growth

\footnotetext{
* This work was performed under the auspices of the U.S. Department of Energy by University of California Lawrence Livermore National Laboratory under contract No. W-7405-Eng-48, and by University Lawrence Berkeley National Laboratory under contract AC0376 SF00098.
}

rate proportional to the beam current. The latter is produced by the combination of chromatic aberration of the system together with transverse misalignment of the solenoidal guide field. Both effects degrade the effective beam spot size and must be limited in magnitude.

This paper discusses the means by which we plan to control BBU and corkscrew growth in DARHT-II. In section 2 we present the current design for the solenoidal field tune; since the last PAC meeting in 1999, the design beam current has been lowered from 4 to $2 \mathrm{kA}$ which has lowered the necessary field strengths. In Sec. 3 we discuss the present predictions for the expected BBU growth; these predictions were made having used recent experimental measurements for the impedance of the DARHT-II accelerator cells. Finally, in Sec. 4 we present our most recent calculations for the expected corkscrew growth and also the expected performance of the "tuning-V" algorithm, which can reduce this growth by more than an order of magnitude.

\section{THE DARHT-II LATTICE AND TUNE}

The DARHT II accelerator lattice may be subdivided into three sections. Referring to Fig. 1, the first section begins just beyond the anode at $Z=100 \mathrm{~cm}$ and consists of 8 "injector" accelerator cells with a 14" beam pipe diameter. These cells produce a nominal $175 \mathrm{kV}$ voltage and each has both a solenoidal magnet with a peak field capability of $\sim 1.0 \mathrm{kG}$ (limited by cooling water considerations) and dipole correction magnets. Beginning at about $550 \mathrm{~cm}$ is the "beam clean-up zone" (BCUZ) which consists of a few solenoids and two apertures. The BCUZ, designed by LANL and Mission Research Corp., produces a highly chromatic tune and has the purpose of removing (via scraping on the apertures) the great majority of off-energy particles produced in the beam head and tail regions (due to the slowly rising injector voltage pulse). Downstream of the BCUZ are the remaining ( $70-80$ in number depending upon final machine configuration) "standard" accelerator cells, each producing $193 \mathrm{kV}$ of energy increase together with a solenoidal magnet with a peak field capability of $\sim 1.6 \mathrm{kG}$ and a set of dipole correction magnets. The standard cells have a 10 " beam pipe diameter -- the change from the injector cells has a positive effect in detuning resonant BBU frequencies. The standard cells are arranged in groups of 6 separated by a short intercell region containing diagnostics and pumping. In addition, the first 4 intercells beyond the BCUZ also have a solenoidal magnet whose purpose is minimize any jumps in the beam envelope $R^{\prime}$ and $R$. The lower panel of Fig. 1 shows the predicted behavior of the beam radius with this 
tune. One sees that apart form the BCUZ region, there is extremely small flutter in $R^{\prime}$ and $R$ which should aid the struggle to maintain beam quality.
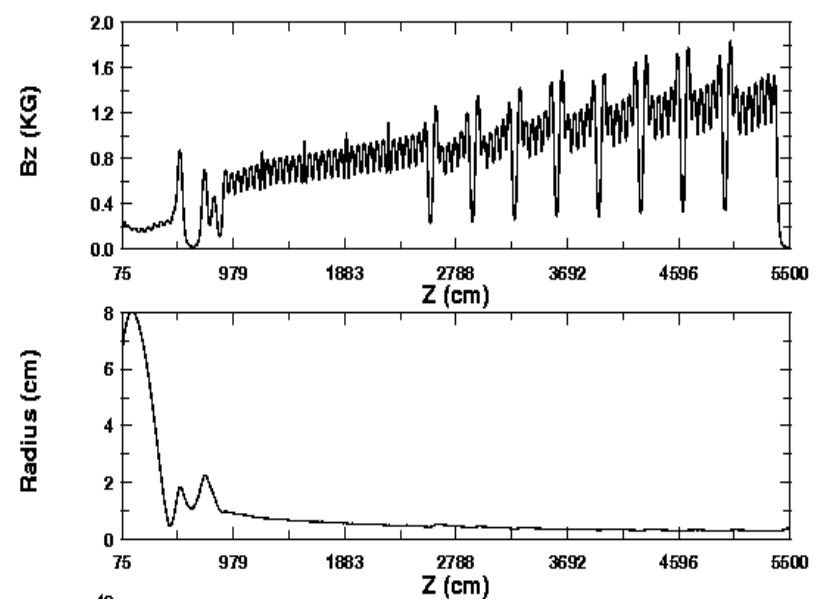

Fig. 1 The upper panel shows the desired Bz tune versus $\mathrm{z}$ for a 2-kA beam current. The lower panel shows the resulting $\mathrm{R}(\mathrm{z})$.

\begin{tabular}{|c|c|c|c|c|}
\hline $\begin{array}{c}\text { Excitation } \\
\text { Frequency } \\
(\mathrm{MHz})\end{array}$ & $\begin{array}{c}\mathrm{Z}_{\perp} \\
(\mathrm{ohm} / \mathrm{m})\end{array}$ & $Q$ & $\begin{array}{c}\text { Amplitude } \\
(\mathrm{mm}) \\
\text { @ cell 44 }\end{array}$ & $\begin{array}{c}\text { Amplitude } \\
(\mathrm{mm}) \\
\text { @ cell 88 }\end{array}$ \\
\hline 146 & 106 & 4.0 & 0.125 & 0.11 \\
\hline 200 & 120 & 2.75 & 0.14 & 0.16 \\
\hline 500 & 500 & 2.0 & 0.125 & 0.115 \\
\hline 168 & 93 & 15 & 0.145 & 0.155 \\
\hline 236 & 251 & 2.5 & 0.16 & 0.225 \\
\hline 572 & 278 & 5.9 & 0.17 & 0.26 \\
\hline ALL-full $\mathrm{Z}_{\perp}$ & & & 0.145 & 0.18 \\
\hline ALL-no $\mathrm{Z}_{\perp}$ & & & 0.13 & 0.105 \\
\hline
\end{tabular}

Table 1 The predicted BBU amplitude as a function of RF parameters of the DARHT-II cell.

\section{BEAM BREAK-UP INSTABILITY}

A quite extensive experimental and computational campaign was conducted to lower both the transverse resistive impedance $Z_{\perp}$ and the cavity $Q$ in order to limit BBU growth [2]. The resulting measurements were then used as input to the LLNL BREAKUP code, together with the lattice $B_{z}$ and $\gamma(z)$ profiles to compute the predicted BBU response. As shown in Table 1, we presumed initial excitations on the level of $100 \mu \mathrm{m}$ (the maximum permitted level according to the injector specification) for each of the resonant BBU frequencies in the injector and standard cells. The final displacements are on the order of $0.2 \mathrm{~mm}$ or less which is acceptably small compared with the DARHT-II goal of $0.1 R$ or $\sim 0.3 \mathrm{~mm}$. We also computed growth for a simultaneous excitation at the 50$\mu \mathrm{m}$ level for all BBU resonant frequencies -- here too the growth (the second to last row of Table 1) remains below the wanted level. The last row of Table 1 shows the predicted displacement level for the same $50-\mu \mathrm{m}$ excitation with $Z_{\perp}=0$ indicating the actual cell impedances lead to no more than a factor of $\sim 2$ net growth. This small value can be attributed to both the steadily rising $B_{z}$ and the strong resonant frequency detuning between the injector and standard cells.

\section{CORKSCREW MOTION AND TUNING- V ALGORITHM}

In order to meet the radiographic performance, the amplitude of the DARHT-II transverse beam motion at the accelerator exit needs to be less than $10 \%$ of the beam radius, i.e., $\leq 0.5 \mathrm{~mm}$. The DARHT-II magnets' alignment specification for tilt and offset in each plane is $2.1 \mathrm{mrad}(3-\sigma)$ and $2.8 \mathrm{~mm}(3-\sigma)$, respectively. BREAKUP simulations (1024 Monte Carlo runs) using the specification for tilts alone and $\pm 0.5 \%$ energy variation predict the corkscrew amplitude to be $0-2.5 \mathrm{~mm}$ and the average beam centroid to be $0-8 \mathrm{~mm}$ without any corrective measures. Similarly, 1024 Monte Carlo runs with the offset specification along predict the uncorrected corkscrew amplitude to be $0-1.5 \mathrm{~mm}$ and the average uncorrected centroid to be $0-6 \mathrm{~mm}$. In order to meet the specification for the beam motion, minimizing the beam motion by steering is needed.

\subsection{Tuning-V Algorithm}

An electron beam is an undamped harmonic oscillator. Its corkscrew amplitude in a misaligned transport system is linearly proportional to the Fourier component of the error transverse field normalized to the $B_{z}$ field at $k=k_{c}$ [3], where $k_{c}$ is the cyclotron wavenumber. The tuning- $\mathrm{V}$ steering algorithm utilizes the fact that any given steering coil pair should have its unique optimal setting to provide the needed $k_{c}$ component to cancel the error field's $k_{c}$ component, presuming unlimited power supply for the steering coil. The $k_{c}$ component of the net transverse field vanishes so that corkscrew amplitude is minimized. To find this unique current setting, we sweep the steering coil's current and measure the time averaged corkscrew amplitude over the flattop portion of the beam current at a downstream beam position monitor (BPM) for each steering coil's current setting. The V-shaped tuning curve for the time average corkscrew amplitude versus the steering coil's current is parabolic near its minimum, which defines the optimal steering coil setting. We then repeat the procedures with a steering coil in the other plane. If the resulting corkscrew amplitude is not small enough, we can repeat the same steps again with other steering coil pairs. The tuning-V algorithm has demonstrated an order of magnitude reduction in corkscrew on the ETA-II accelerator [5] and the Flash X-ray Radiography accelerator (FXR) [6].

The DARHT-II accelerator consists of $78-88$ cells, depending on the machine configuration, with one BPM per 8 cells and one pair of steering coils per cell. The machine will deliver an electron current pulse every $1-5$ 
minutes and approximately 500 shots per week. If the accelerator is steered iteratively, starting at the injector and sequentially adjusting the current in each steering coil for a minimum in the time averaged corkscrew amplitude observed by a downstream BPM until the end of the accelerator is reached, it would take at least a week to complete the steering. Our experience on ETA-II and FXR and on BREAKUP simulations indicates that not all steering coils can minimize the corkscrew motion at a given BPM efficiently. Therefore, to save the valuable shots, we should start the steering procedure by choosing the "efficient" steering coils first. Fire 3 shots with a given steering coil set at 0 and $\pm I_{\max }$ which is the maximum available current for the steering coil. If there is no noticeable variation in the observed corkscrew amplitudes among these three shots, don't use this steering coil. Otherwise, fit the 3 data points with a parabolic. Then depending on the curve, either set the steering coil at $I_{\max }$ or - $I_{\max }$, or chose a range of steering coil current to get a tuning curve. Furthermore, it is not necessary to minimize the corkscrew motion at every BPM even though there is one BPM for every 8 cells. As long as the cyclotron wavenumber does not vary too much over a distance, we can always use several steering coils to provide a net correction field such that its Fourier component at $k_{c}$ cancels the misalignment field's Fourier component. Therefore, we only need to minimize the corkscrew motion at the BPM at the end of this distance.

\subsection{BREAKUP Simulations}

We have simulated the corkscrew motion on the DARHT-II accelerator with two tuning schemes. The simulations include $\pm 0.5 \%$ energy sweep on the injector and gap voltages, and a large injector offset $(\sim 1 \mathrm{~mm})$ and tilt $(\sim 1 \mathrm{mrad})$ introduced by the dipole field in the DARHT-II injector configuration. Without any steering, the BREAKUP simulation predicts that the corkscrew amplitude at the accelerator exit is about 8 times of the design specification. Let $R$ be the averaged centroid radial displacement over the flattop portion of the current pulse, and $A$ be the averaged corkscrew amplitude. For the first tuning scheme, the tuning- $\mathrm{V}$ steering algorithm is used to minimize a figure of merit $M$, where $M=\left(W^{*} R\right)^{2}+A^{2}$, and $W$ is a weighting factor, at every BPM with $W=0.1$. For the second tuning scheme, only 2 BPMs are used. Corkscrew motion is minimized at the BPM after Block 8 . Then, it is minimized again at the end of accelerator by using the steering coils right after Block 8 . Finally, the centroid at the exit is minimized by using the steering coils at the end of the accelerator. As shown in Table 2, the final corkscrew amplitude and beam centroid displacements of all cases are very similar. These results suggest that minimizing the number of tuning shots by tuning the corkscrew motion only at a few BPMS will meet the accelerator performance.

\begin{tabular}{|c|c|c|c|}
\hline $\begin{array}{c}\text { Tuning } \\
\text { Scheme }\end{array}$ & Every BPM & Every BPM & 2 BPMs \\
\hline Cases & 3 & 1 & 3 \\
\hline $\begin{array}{c}\text { Maximum } \\
\text { Field per } \\
\text { Steering Coil }\end{array}$ & 5 Gauss & 8 Gauss & 5 Gauss \\
\hline Coils Used & $46-60$ & 35 & $16-34$ \\
\hline Shots Used & $300-424$ & 294 & $83-137$ \\
\hline $\begin{array}{c}\text { Time Average } \\
\text { Corkscrew }\end{array}$ & $\begin{array}{c}0.16-0.24 \\
\mathrm{~mm}\end{array}$ & $0.15 \mathrm{~mm}$ & $\begin{array}{c}0.1-0.17 \\
\mathrm{~mm}\end{array}$ \\
\hline $\begin{array}{c}\text { Beam } \\
\text { Centroid }\end{array}$ & $\begin{array}{c}0.23-1.4 \\
\mathrm{~mm}\end{array}$ & $0.8 \mathrm{~mm}$ & $\begin{array}{c}0.22-2 \\
\mathrm{~mm}\end{array}$ \\
\hline
\end{tabular}

Table 2 Comparison of two different tuning-V schemes

\section{SUMMARY}

Our study suggest that BBU growth in the DARHT-II should not seriously degrade the final achievable spot size at the $\mathrm{x}$-ray converter, presuming the initial BBU excitation level is of the order 100 microns or smaller. Performance of the tuning- $\mathrm{V}$ steering algorithm depends on shot-to-shot repeatability of the machine. Our study suggest that controlling corkscrew motion in the accelerator with the tuning- $\mathrm{V}$ steering algorithm could be achieved, presuming the solenoid magnet alignment falls within the design specifications, and the machine is repeatable. As the DARHT-II facility may be limited to approximately 500 shots per week, it is quite significant (and possibly necessary) that the number of shots needed to implement the tuning- $\mathrm{V}$ algorithm can be possibly as few as $50-100$ by minimizing the time average corkscrew amplitude at 2 BPMS only, providing the "efficient" steering coils have already been identified. At the initial setup stage of a new magnetic tune, more shots will be needed to identify the "efficient" steering coils which minimize corkscrew motion observed by a chosen BPM.

\section{REFERENCES}

[1] M. Burns et al., "Status of the DARHT Phase 2 Long-Pulse Accelerator", Paper AAA, this Proceedings.

[2] R. Briggs et al., "Transverse Impedance Measurements of the DARHT-2 Accelerator Cells", Paper BBB, this Proceedings.

[3] Y.-J. Chen, Nucl. Instr. Meth. A 398, 139 (1997).

[4] "DARHT", LANL Report No. LALP 99-1414, August 1999.

[5] J. Weir et al., "Improved ETA-II Accelerator Performance", Proc. 1999 Particle Accel. Conf,, New York, NY, 3513 (1999).

[6] R. Scarpetti and J. Zentler, private conversation. 